\title{
DIRECTORY OF IUGS BODIES
}

\section{COMMISSIONS}

COMMISSION ON COMPARATIVE PLANETOLOGY (CCP)

Secretary General: Dr. R.A.F. Grieve, Geological Survey of Canada,

1 Observatory Crescent, Ottawa, Canada KIA $0 Y_{3}$

COMMISSION ON GEOLOGICAL DOCUMENTATION (COGEODOC)

Secretary-Treasurer: Dr. J. Gravesteijn, Lépt. Doc/BRGM, BP 6009, $\mathrm{F}-45018$ Orléans-Cedex, France.

COMMISSION ON GEOLOGY TEACHING (CGT)

Chairman: Prof. W.H. Watthews, III, Box 10031, Lamar University,

Beaumont, Texas 77710 , U.S.A.

COMMISSION ON GLOBAL SEDIMENTARY GEOLOGY

Chairman: Lr. R.N. Ginsburg, Fisher Island Station, University of Miami,

Iiami Beach, FL 33139 , U.S.A.

COMMISSION ON THE HISTORY OF GEOLOGICAL SCIENCES (INHIGEO)

Genergl Secretary: Dr. E. Dudich, Division of Earth Sciences, UNESCU,

Place de Fontenoy, B.P. 3.07, F-75700 Paris, France.

COMMISSION ON IGNEOUS AND METAMORPHIC PETROGENESIS (CIMP)

Chairman: Prof. A.J. Naldrett, Lepartment of Geology, University of

Toronto, Toronto, Canada, M5S IAl.

COMMISSION FOR MARINE GEOLOGY (CMG)

Secretary: Prof. J. Thiede, Department of Geology and Palaeontology, Kiel University, Olshausenstr. 40/60, 1-2300 Kiel, F.R.G.

COMMISSION ON STORAGE, AUTOMATIC PROCESSING AND RETRIEVAL OF GEOLOGICAL DATA (COGEODATA)

Secretary-Treasurer: Dr. G. Gabert, Federal Institute for Geosciences and Natural Resources, P.O. Box 510153, D-3000 Hannover 51, Federal Republic of Germany.

COMMISSION ON STRATIGRAPHY (ICS)

Chairman: Dr. J.W. Cowie, Department of Geology, University of Bristol, linistol BS8 1TR, U.K.

COMMISSION ON SYSTEMATICS IN PETKOLOGY

Chairman: Dr. P.A. Sabine, 19 Beaufort koad, Hanger Hill, Ealing, London W. $3 \mathrm{~EB}$, U.K.

COMMISSION ON TECTONICS (COMTEC)

Secretary: Prof. A. Kröner, Johannes Gutenberg-Universität wainz, Postfach 3980, Saarstrasse 21, 6500-Mainz, F.R.G.

\section{ADVISORY BOARDS}

ADVISORY BOARD FOR RESEARCH DEVELOPMENT

Chairman: Dr. R.W.R. Kutiand, Bureau of ivineral Resources, Geology and Geophysies, P.O. Box 378, Canberra, A.C.T. 2601, Australia.

ADVISORY BOARD FOR PUBLICATIONS

Chairman: Dr. J. Aaron, U.S. Geological Survey, uffice of Scientific

Publications, MS904, National Center, Reston VA 22092, U.S.A.

ADVISORY BOARD ON REMOTE SENSINC

hairman: Dr. P. Teleki, U.S. Geological Survey, NS917, National Center, Reston, VA 22092, U.S.A.

\section{COMMITTEES}

COMMITTEE ON SEDIMENTOLOGY

Secretary-Treasurer: Dr. R.N. Ginsburg, Fisher Island Station, University of Viami, Miami Beach, FL 33139, U.S.A.

ADVISORY COMMITTEE ON THE EVALUATION OF FOSSIL FUELS Chairman: C. Salle, IF P, 1 A4 Av. de Bois-Preault, 92506 Rueil Naimaison, France.

WORKING GROUP ON PETROLEUM RESOURCE ASSESSMENT

hairman: Lr. C.D. Nasters, U.S. Geological Survey, ils955, National

Center, Reston, VA 22092, U.S.A.

\section{AFFILIATED ORGANIZATIONS}

ASSOCIATION OF ARAB GEOLOGISTS (AGA)

Secretary General: Dr. Sammi Sheriff, Iraq National Oil Company, Al-Khulani Sq., Baghdad, Iraq.

ASSOCIATION OF EXPLORATION GEOCHEMISTS (AEG)

Secretary: Dr. K.E. Lett, P.O. box 523 , Rexdale, Ontario, Canada M9W 5 L 4 . ASSOCIATION OF GEOSCIENTISTS FOR INTERNATIONAL

DEVELOPMENT (AGID)

Secretary-Treasurer: Lr. Theerapongs Thanasuthipitak, c/o AGID, Asian Institute of Technology, Box 2754, Bangkok 10501, Thailand.

ASSOCIATION INTERNATIONALE POUR L'ETUDE DES ARGILES (AIPEA) Secretary General: Dr. A.J. Herbillon, Université Catholique de Louvain, Groupe de Physico-Chimie Minérale et de Catalyse, Place Croix du Sud 1 , 1348 Louvain-La-Neuve, Belgium.
ASSOCIATION DES SERVICES GEOLOGIQUES AFRICAINS (ASGA) Secretary-General: Dr. L. Delbos, 103, rue de Lille, 75007 Paris, France.

CARPATHIAN BALKAN GEOLOGICAL ASSOCIATION (CBGA)

President: Dr. Ing. I. Folea, Wlinistère de la Géologie, str. Wendeleev, 34-36, Bucharest, kumania.

CIRCUM-PACIFIC COUNCIL FOR ENERGY AND MINERAL RESOURCES President and Chairman: Michel T. Halbouty, The Halbouty Center, 5100 Westheimer Road, Houston, Texas 77056, U.S.A.

COMMISSION FOR THE GEOLOGICAL MAP OF THE WORLD (CGMW) Secretary-General: O.Y.J. Dottin, Maison de la Géologie, 77 , rue Claude Bernard, 75005 Paris, France.

EUROPEAN ASSOCIATION OF SCIENCE EDITORS (EASE)

Secretary-Treasurer: Ms. Maeve O'Connor, 13 wimpole Street, London WIM 7AB, U.K.

GEOLOGICAL SOCIETY OF AFRICA (GSA)

Secretary-General: F.G. Theuri, Wines and Geological bepartment, P.O. Box 30009 , Nairobi, Kenya.

INTERNATIONAL ASSOCIATION OF ENGINEERING GEOLOGY (IAEG) Secretary General: Dr. W. Primel, L.C.P.C., 58, Bd. Lefebvre, 75732 Paris Cedex 15 France.

INTERNATIONAL ASSOCIATION OF GEOCHEMISTRY AND COSMOCHEMISTRY (IAGC)

Secretary-General: Dr. B. Hitchon, Alberta Research Council, Terrace Plaza 4445 Calgary Trail South, Edmonton, Alberta Canada T611 $5 \mathrm{R} 7$.

INTERNATIONAL ASSOCIATION ON THE GENESIS OF ORE DEPOSITS (IAGOD)

Secretary General: Prof. i. Vanecek, Department of teonomic Geology, Charles University, Albertov 6, 12000 , Praha 2, Czechoslovakia. Associat Secretary General: Dr. I. Jonasson, e/o Geological Survey of Canada, 601 Booth St., Uttawa, Canade KlA 0t8.

INTERNATIONAL ASSOCIATION OF HYDROGEOLOGISTS (IAH) Secretary General: E. Komijn, Prov. Waterboard of Gelderland, Warkstraat 1, P.O. Box 9090 , NL-6800 GX Arnhem, The Netherlands.

INTERNATIONAL ASSOCIATION FOR MATHEMATICAL GEOLOGY (IAMG) Secretary General: Dr. K.B. VleCammon, U.S. Geological Survey, National Center 920, Reston, VA 22092 , U.S.A.

INTERNATIONAL ASSOCIATION OF PLANETOLOGY (IAP)

Secretary: Dr. N. Stovickova, Institute of Applied Geophysics, Podbelohorska 47, 15000 Praha 5, Czechoslovakia.

INTERNATIONAL ASSOCIATION OF SEDIAENTOLOGISTS (IAS)

Secretary-General: Wr. F. Surlyk, Geological Survey of Greenland, Uster Voldgade 10, Copenhagen DK-1350, Denmark.

INTERNATIONAL FEDERATION OF PALYNOLOGICAL SOCIETIES (IFPS) Secretary-Treasurer: Dr. David M. Jarzen, National viuseum of Natural Sciences, Paleobiology Division, 1767 Woodward Drive, Ottawa, Canada KIA 0 M 8 .

INTERNATIONAL MINERALOGICAL ASSOCIATION (IMA)

Secretary-General: Dr. S.S. Hafner, Institute of vineralogy, University of Marburg, Lahnberge, 3550 Marburg, F.R.G.

INTERNATIONAL PALAEONTOLOGICAL ASSOCIATION (IPA)

Secretary-General: William A. Oliver, Jr., U.S. Geological Survey, E-305 National Museum Building, Washington, DC 20560 , U.S.A.

INTEKNATIONAL UNION FOR QUATERNARY RESEARCH (INQUA) Secretary-Treasurer: Ch. Schlüchter, Institute of Foundation Engineering, ETH-Hönggerberg, CH-8093 Zurich, Switzerland.

SOCIETY OF ECONOMIC GEOLOGISTS (SEG)

Secretary: Dr. J.F. Nurphy, P.O. Box 571, Golden, Colorado 80402, U.S.A. SOCIETY FOR GEOLOGY APPLIED TO MINERAL DEPOSITS (SGA) Secretary: Lr. F. Saupé, Centre de Recherches Pétrographiques et Géochimique, 15, rue N.-L, des Pauvres, 54501 Vandoeuvre, Les Nancy Cedex, France.

INTERNATIONAL GEOLOGICAL CORRELATION PROGHAMME (IGCP) Secretary: Dr. E. Dudich, IGCP, Division of Earth Sciences, UNESCU, Place de Fontenoy, B.P. 3.07, F-75700 Paris, France.

INTER-UNION COMMISSION ON THE LITHOSPHERE (ICL) Secretary General: Prof. H.L. Zwart, Instituut voor aarawetenschappen, Kijksuniversiteit Utrecht, Budapestlaan 4, Postbus 80.021, 3508 TA Utrecht, The Netheriands.

28th INTERNATIONAL GEOLOGICAL CONGRESS (IGC), 1989

Secretary General: Dr. B. Hanshaw, International Geological Congress, P.U. Box 1001 , Herndon, VA 22070 , U.S.A. 